\title{
Circuit
}

Musiques contemporaines

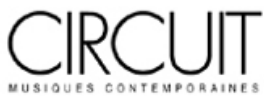

\section{Le charme étrange des espaces familiers}

Périple analytique dans Treppenumusik de John Rea

The Strange Fascination of Familiar Spaces

\section{An Analytical Voyage Through John Rea's Treppenmusik}

\section{Michel Gonneville}

Volume 11, numéro 1, 2000

\section{Analyses}

URI : https://id.erudit.org/iderudit/004674ar

DOI : https://doi.org/10.7202/004674ar

Aller au sommaire du numéro

Éditeur(s)

Les Presses de l'Université de Montréal

ISSN

1183-1693 (imprimé)

1488-9692 (numérique)

Découvrir la revue

Citer cet article

Gonneville, M. (2000). Le charme étrange des espaces familiers : périple analytique dans Treppenumusik de John Rea. Circuit, 11(1), 8-32.

https://doi.org/10.7202/004674ar

\section{Résumé de l'article}

Fasciné par Treppenmusik de John Rea, l'auteur en explore les diverses facettes, entre autres celle de l'harmonie, où l'emploi d'éléments historiquement chargés se conjugue à des techniques qui laissent peu de place à la nostalgie et renouvellent le sens de ces « choses connues ». Il cherche ensuite à dégager certaines directions formelles générales de l'oeuvre. Finalement, et plus généralement, il interroge le futur du postmodernisme, dont Treppenmusik lui semble un exemple achevé. 

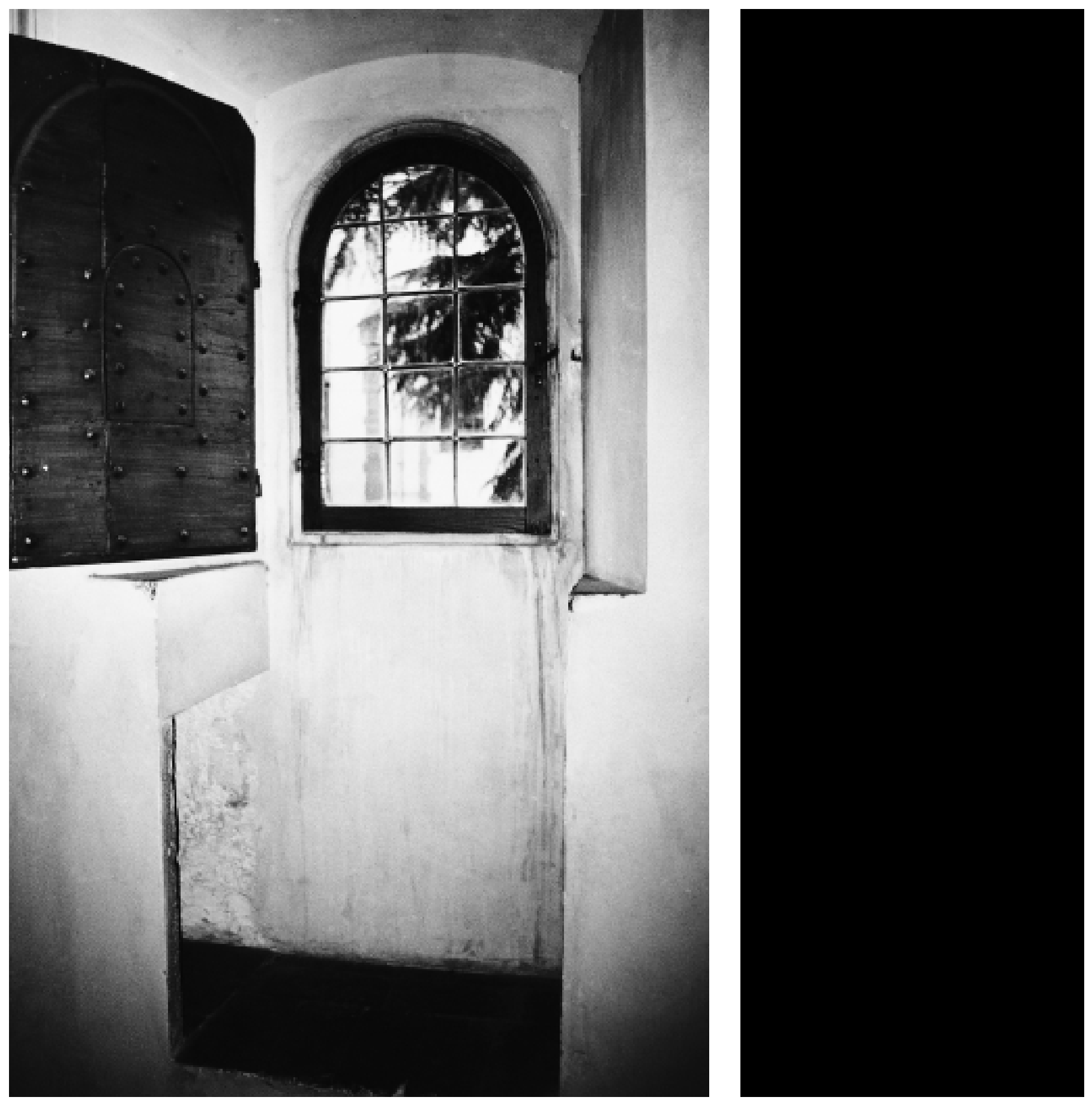


\title{
Le charme étrange des espaces familiers \\ Périple analytique dans Treppenmusik de John Rea
}

\author{
$M$ ichel $G$ onneville
}

D'ailleurs, l'analyse musicale ne fait qu'éclairer la jouissance.

ChARLES Rosen, 1993, p. 63.

Treppenmusik de John Rea m'a toujours fasciné. Cette œuvre écrite en 1982 me semble l'une des belles réussites de la postmodernité québécoise, également illustrée par des compositeurs comme Claude Vivier, José Evangelista, Denis Gougeon, etc. Et puisque ce courant est souvent (et de façon trop simpliste) associé à la citation, campons le propos de cet article à l'aide d'une citation de John Rea (sertie elle-même - mise en abîme... - d'une autre citation de Claude Vivier): il s'agit de la notice figurant au début de la partition de l'œuvre ${ }^{1}$ :

Treppenmusik (musique dans l'escalier) est le motallemand qui qualifie Sieg fried -Idyll de W agner. En effet, la première exécution de cette œuvre, cadeau de $\mathrm{N}$ oël et d'anniversaire pour C osima, eut lieu dans l'escalier de W ahnfried, une des villas de W agner. Dans ma composition toutefois, ce titre fait allusion aux escaliers paradoxaux - montées et descentes vers quelque part / nulle part - ainsi qu'aux autres boucles étranges figurant dans les gravures et lithographies de l'artiste hollandais M aurits Cornelis Escher (1898-1972).

Par ses expériences avec la perspective et les agencements en mosaïque, Escher a réuni dans son œuvre symétries, répétitions à caractère canonique, métamorphoses, mutation du familier en fantastique - techniques et principes de base qui subissent également l'influence des ma thématiques. II semble vérita blement avoir créé par des formes irrésistibles un autre univers de sensibilité régi par sa propre mécanique céleste, aussi impossible soitelle.

1. Cette partition, photocopie du manuscrit du compositeur, est disponible au Centre de musique canadienne au $\mathrm{Q}$ uébec.

Treppenmusik, pour 12 instruments et système de délai par ruban magnétique, a été écrit entre mai et août 1982, à Montréal. Il s'agit d'une commande de la Société de musique contemporaine du $\mathrm{Q}$ uébec. L'œuvre est dédiée à cette société et a été créée par l'ensemble de la Société de musique contemporaine du $Q$ uébec (SM C Q ) sous la direction de son directeur musical, Serge G arant L'œuvre figure sur le CD M ontréal Postmoderne de la Société nationale d'enregistrement. 
Comme l'écrivait récemment mon cher a mi Vivier: "Le temps est un espace à géométrie variable. Les différents plans se côtoient, se pénètrent et se transforment suivant les règles merveilleuses de la mécanique céleste... La musique engendre la magie du temps dans la vie humaine. Pour quelques courts instants, l'être humain transgresse le grand ordre de la méca nique céleste... C'est ainsi que l'humain construit ses machines à voyager dans le temps: la musique. » («Pour G ödel», dans Trafics, M ontréal, 1982).

Treppenmusik est ma propre machine à voyager dans le temps, à l'intérieur d'un monde selon Escher.

Dans cette double citation se révèlent certains traits distinctifs de la personnalité de Rea et de Vivier. O $\mathrm{n}$ reconnaît le besoin du premier de «faire référence à », d'assumer, de commenter et de porter plus loin une culture historique et artistique, et ce, non seulement dans des écrits et conférences mais jusque dans le propos des œuvres musicales. Ici, la musique tisse des liens avec sa propre culture, bien sûr, mais aussi avec le domaine des arts visuels, comme dans Hommage à Vasarely ou Spin, deux autres œuvres de Rea. De plus, l'appropriation de la citation de Vivier, a vec sa référence à la magie et au merveilleux, à la transgression du réel, ne doit pas laisser croire à une trop grande parenté entre les deux compositeurs, tant sur le plan de la thématique d'inspiration que sur celui de la visée expressive et dramatique. Alors que le voyage dans le temps prend, pour Vivier, la signification d'une fuite, d'un oubli du Temps et de la vie limitée afin de s'ouvrir à d'autres plans, ceux d'un imaginaire ressourçant, d'une Autre Vie imaginée et désirée; pour Rea, il s'agira au contraire de prendre avec soi le Temps, l'héritage culturel, le passé a vec tout son poids et de l'amener sur d'autres socles où le considérer, sous d'autres éclairages, de faire subir à ce Temps des métamorphoses pour le transmuer en fantastique, en inouï. II est révélateur aussi que cette notice de Rea attire notre attention sur le comment, sur les procédés plus que sur une intention expressive, et qu'elle se serve des mots sortis de la plume de Vivier pour parler de magie et de merveilleux... Est-ce là pudeur? Car la magie qui naît de ce jeu de références transgressées, bien que d'un autre ordre, est pourtant bien réelle.

\section{Temps (choses connues)}

J'ai proposé, dans un article paru précédemment (G onneville, 1990), une définition du postmodernisme musical fondée sur l'aspect concret du matériau utilisé plutôt que sur les réflexions sociologiques, politiques ou psychanalytiques qui en justifieraient la position esthétique. Je l'ai associé à la réintégration d'éléments de langage que le modernisme musical «pur et dur» ava it ou s'était interdit (périodi- 
cité, répétition, thématisme, consonances, etc.). Il s'a girait, pour ce courant, d'entendre d'une oreille neuve ces choses connues, provenant d'un passé plus ou moins lointain, non seulement en les citant au sein de matériaux plus récents ou, plus subtilement, en composant des allusions stylistiques, mais aussi et surtout en articulant ces éléments familiers selon des règles syntaxiques et formelles inattendues, qui, elles, peuvent être influencées par les techniques issues de l'a vant-ga rde.

Si l'affirmation (déjà ancienne) de M essiaen, selon laquelle plus rien ne peut être trouvé sur le plan harmonique, parce que nous aurions «atteint un plafond», si cette a ffirmation est peutêtre vraie en ce qui concerne l'accumulation [du matériel harmonique], elle ne l'est certainement pas, il s'en faut, en ce qui concerne la compréhension. Autrement dit, si nous avons appris à accepter pratiquement tout ce qui peut se produire [sur ce même plan harmonique], nous sommes bien loin d'avoir appris ce que virtuellement cela signifie, tout ce qui syntaxiquement peut en être fait; nous sommes même sur ce plan encore extrêmement myopes, extrêmement maladroits ${ }^{2}$.

(Henri Pousseur, 1968, p.150.)

Le postmodernisme critique dont se réclame Rea ne se contentera pas de nous ramener en arrière, de se réfugier dans ces choses connues «parce que le modernisme était un cul-de-sac »; il cherchera plutôt à nous les faire voir sous une lumière nouvelle. $0 \mathrm{n}$ pourrait dire que John Rea adopte l'attitude exploratoire, «expérimentale » du modernisme en ce sens qu'il ne traite pas le donné comme une évidence ou une vérité destinée à «l'expression» convenue d'émotions, mais plutôt comme une curiosité, un objet de questionnement. II manipule et redécouvre le matériau musical connu et familier, nous le fait redécouvrir, lui donne un "sens » nouveau, ce nouvel agencement d'énergies musicales stimulant de nouvelles zones de l'imaginaire et suscitant de nouvelles émotions.

Dans Treppenmusik, les choses connues sont de plusieurs ordres et de degrés de reconnaissance variables. Au premier degré, il y a la citation pure et simple. Ainsi les mesures 282 à 296 nous présentent un extrait du «Jeu du rapt» tiré du Sacre du printemps d'Igor Stravinski, très proche de l'original sauf évidemment en ce qui concerne la couleur orchestrale. $0 \mathrm{n}$ entendra aussi, dès la sixième mesure et de façon répétée pendant toute la section introductive (exemple 1$)^{3}$, le motif du $\mathrm{N}$ ibelung (le martèlement des forgerons), tiré de l' $\mathrm{r}$ du Rhin de $\mathrm{W}$ agner, légèrement modifié dans sa courbe mélodique. Hormis le titre, ce motif sera d'ailleurs la seule référence explicite à $W$ agner. Beaucoup plus nombreuses et intéressantes, parce que plus subtiles et ambiguës, sont les allusions stylistiques que l'on peut relever au fil de l'œuvre. Le paramètre harmonique joue un rôle fondamental dans la perception de ces allusions. Ainsi, la manipulation d'accords faits de quintes superposées ou l'emploi de la gamme par tons eng endrent des harmonies modales aux couleurs debussystes. Ailleurs, les quartes superposées ne peuvent manquer d'évoquer Schönberg. Et de même, l'inclusion d'un accord qui reproduit la série des ha rmoniques na turels nous renvoie, au-delà de l'identification d'un archétype, à la musique spectrale des $\mathrm{G}$ risey et $\mathrm{M}$ urail. La mise en temps de ces harmonies
2. Les additions entre crochets sont de l'auteur.

3. Pour la définition des sections, on se reportera au tableau de la fin de l'article. 
se fait sous forme de tournures motiviques ou de figurations familières déployées dans un contexte de continuité rythmique, de référence à une pulsation ou à une métrique constantes - ce qui, à l'époque de la création de Treppenmusik, était encore plus ou moins un tabou dans le monde de la musique contemporaine. Tous ces éléments, ces harmonies et ces manières orientent donc notre perception, une fois le quoi reconnu, vers le comment de leur emploi. Et c'est dans ce comment que réside la spécificité postmodeme. John Rea me disait qu'à l'exemple du Centre $\mathrm{G}$ eorges-Pompidou du plateau Beaubourg à Paris, dont la structure se retrouve à l'extérieur, montrée, le postmodernisme musical dévoile les processus. $0 \mathrm{n}$ pense évidemment à la musique répétitive qui a voulu prendre le contre-pied de l'abstraction des néosériels et rendre évidents les processus musicaux. John Rea est près de cette attitude sans adhérer cependant au minimalisme - fût-il choisi et nécessaire - qui a caractérisé ce courant à ses débuts.

\section{Espaces colorés, dimension I: timbres, distribution}

Approfondissons ces quelques généralités.

John Rea a choisi pour Treppenmusik l'instrumentation suivante : 4 saxophones (1 soprano, 1 alto, 1 ténor, 1 baryton), 4 clarinettes (1 en mi bémol, 1 en si bémol, 2 basses) et 4 cordes différentes (1 violon, 1 alto, 1 violoncelle, 1 contrebasse). Les saxophones, quasi-cuivres, ont sans doute été choisis en raison de leur virtuosité, comparable à celle des clarinettes et des cordes. Tout l'ensemble est capté par des microphones, et un dispositif simple permet quelques altérations en direct du son, so it la spatialisation sur qua tre hautparleurs situés autour du public, la rétroaction (feedback) et la transposition, traitements utilisés par ailleurs de façon économique et discrète. Malgré cette discrétion, on verra tout de même dans cette addition technologique un moyen supplémentaire de propulser le son d'un instrumentarium somme toute classique vers des zones moins habituelles, d'ajouter des zones de flou à des événements sonores que notre habitude d'écoute de musique instrumentale nous a rendus familiers.

Avec cet ensemble, le compositeur dispose, pour chaque famille instrumentale, des «voix» de soprano, d'alto, de ténor et de basse, pour parler dans les termes de l'écriture à quatre parties. L'œuvre exploitera évidemment très souvent ces divisions, soit en trois familles, soit à qua tre voix: canons ou alternances des familles, répartition d'harmonies à qua tre notes, etc. La disposition symétrique des tro is groupes sur scène en trois rangées, une par famille, les instruments disposés de l'aigu à gauche au grave à droite, corrobore cette double conception. $M$ ais une autre 
logique distributive particulière est aussi utilisée très fréquemment, faisant s'interpénétrer registres et familles. Elle répartit la to talité des instruments de l'aigu au grave, les extrêmes de la famille des cordes embrassant ceux de la famille des clarinettes qui embrassent eux-mêmes ceux de la famille des saxophones.

$\begin{array}{rlll}1 & \text { Violon } & \text { Clarinette en mi b } & \\ 2 & & & \\ 3 & & \\ 4 & \text { Alto } & \text { Clarinette en si b } & \\ 5 & & & \text { Sax alto } \\ 6 & & \text { Clarinette basse } 1 & \\ 7 & & \\ 8 & & & \text { Sax ténor } \\ 9 & \text { Violoncelle } & & \\ 10 & & \text { Clarinette basse 2 } & \\ 11 & & & \\ 12 & \text { Contrebasse } & & \end{array}$

Loin d'être purement spéculative ou abstraite, cette distribution timbrale tient compte des limites du registre des instruments: le violon va plus haut que la clarinette en mi bémol qui se rend elle-même plus haut que le saxophone soprano; la contrebasse va plus bas que la clarinette basse qui va elle-même légèrement plus bas que le saxophone baryton, etc. Comme les arrangements par famille ou par registre, cette disposition est elle aussi révélatrice d'une attitude d'esprit, d'une approche "géométrique » du matériau - gardons Escher en tête. $0 \mathrm{n}$ la verra imposer sa symétrie à la logique de succession des événements, à la composition (la mise en temps) du matériau ha rmonico-rythmique et s'offrir comme solution pour l'orchestration des harmonies de haute densité ou très larges, c'està-dire pour la répartition des notes aux différents instruments. Afin d'en saisir I'utilité, on pourra regarder dans la partition: la succession des entrées dans la section $1{ }^{4}$, qui se passent toutes à l'intérieur d'une quarte centrale commune à tous les instruments; l'orchestration des larges accords de quartes superposées dans la section $2 a, 2 b$ et $3 b$; celle de l'accord de série harmonique dans la section 9 (exemple 12); la distribution de la mélodie égrenant le cycle des quintes dans la section $8 \mathrm{a}$. C ette disposition fixe contribue évidemment à la cohérence de la «sonorité » générale de l'œuvre.
4. O n se rapportera au tableau placé en annexe à la fin du texte, qui résume la forme générale de l'œuvre et en définit les sections.

\section{Espaces colorés, dimension II: harmonie}

II peut sembler oiseux de relier le plaisir du vécu musical, ressenti «en temps» bien réel, à un exposé analytique sur le matériau harmonique considéré «hors 
temps». Et pourtant, l'une des dimensions du plaisir musical est liée à cette impression floue, que clarifie l'analyse, d'unité et de variété du matériau, peutêtre surtout sur le plan harmonique qui, entre autres fonctions, donne sa couleur toute particulière à une œuvre ou à un corpus. Survolons donc ce matériau harmonique. La variété de celui-ci pourrait se «réduire » aux familles et aux processus exposés dans les paragraphes suivants.

\section{Q uartes}

Une première famille d'éléments se regroupe autour de l'intervalle de quarte juste que l'on entend dès la première section de l'œuvre (exemple 1 ) et que l'on retrouvera, comme pour boucler le périple, à la fin de la section 19 et au début de la section 20 (exemple 21 ). La deuxième section procède sans attendre à la généralisation de cet intervalle, par expansion sur un registre très étendu jusqu'à un accord de quartes superposées (exemple 2). L'expansion s'arrête juste avant d'atteindre le total des 12 sons (contenu dans un cycle de quartes complet). 0 n retro uve un autre accord de quartes superposées au début du développement par contraction de la section 3b (mes. 98-99, exemple 4), et aussi comme «armature » à la superposition de descentes chromatiques partiellement trillées de la section 7 , a rmature visible, sinon audible, sur les premier et qua trième temps des mesures à $6 / 8$ (exemple 8 ). Bien évidemment, pour cette famille d'éléments, la référence à certains Bartók ou au Schönberg de la Kammersymphonie ne saurait échapper à l'auditeur a verti, même si l'accord de quartes ne peut être considéré comme la propriété exclusive de ces compositeurs, surtout après l'usage qu'en a fait le jazz. De toute façon, ici comme dans les familles qui suivent, le matériau est familier, codé et l'esprit se tend alors vers la manière dont il est utilisé.

\section{Q uintes}

Complémentaires à la famille des quartes, certains éléments se rattachent au renversement de cet intervalle, la quinte juste, et a ux accords qui s'y superposent. Dans une autre œuvre de John Rea, Hommage à Vasarely pour orchestre, I'harmonie était bâtie à partir d'un accord dodécaphonique de quintes superposées, imposé comme une grille fixe pendant toute la durée de l'œuvre; 0 ver Time, également pour orchestre (1987), commence avec une même grille mais «remplie » cette fois par des tierces. 0 n peut supposer que dans Treppenmusik, le choix de la quarte pour bâtir une grille presque dodécaphonique soit en rap- 
port avec le registre des instruments choisis. Q uoi qu'il en soit, la superposition des quintes n'atteint pas ici le total dodécaphonique. Tout a u plus aura-ton un accord de quatre sons qui résulte de trois quintes superposées, utilisé aux sections $2 c$ et $2 d$ (exemple 3 ), ou celui de sept sons (six quintes superposées) entendu dans les sections 5 et 6 (exemples 6 et 7 ). Le cycle complet des quintes sera cependant égrené mélodiquement à cinq reprises pendant la section 8 (avec certains renversements ou «repliements mélodiques » dus parfois aux limites instrumentales) (exemples 9,10 et 11 ). Les harmonies de quintes superposées ont bien sûr déjà été utilisées par Debussy (par exemple dans La cathédrale engloutie) et Bartók (dans le Deuxième Concerto pour piano), et, plus près de nous, Ligeti, dans un esprit très postmoderne, ne s'en est pas privé non plus (Kammerkonzert).

\section{Évidements et remplissages, modes et horizontalisations}

O n peut également rattacher à la fa mille des quartes les accords ou structures de trois septièmes mineures superposées, parce que ces éléments proviennent d'un «évidement» de la structure partielle de quartes, c'està-dire qu'une note sur deux est enlevée. Cette structure de septièmes sert pour les sections 4 - elle est d'ailleurs clairement exposée à la mesure 117 - (exemple 5) et 10 (exemple 14 ). Dans cette dernière section, la sonorité de gamme par tons inhérente à cette superposition intervallique devient évidente (et nous renvoie à Debussy... ) et marquera toute la section 11 qui suit $C$ e sont également des accords de trois sons faits de deux septièmes mineures superposées qui descendent par tons entiers dans la section 14. Complémentaire de cette armature de septièmes et également relié à la gamme par tons, l'accord de trois neuvièmes majeures superposées, provenant de l'évidement d'une armature de quintes, est utilisé lors de la grande «spirale» sans fin de la section 17, tout entière construite sur l'une des deux transpositions de la gamme par tons (les figurations superposées sont à distance de neuvième majeure). II est d'ailleurs révélateur que cette section soit introduite par une courte ascension de quartes, qui montre le lien «théorique » entre ce matériau et la gamme partons.

Les intervalles de quarte ou de quinte peuvent être «remplis» (ou «monna yés», dirait M essiaen) par des notes faisant le lien entre les bornes de ces intervalles. Des figurations mélodiques conjointes peuvent ainsi s'élaborer et l'accord de quarte devient alors, on l'a dit, armature ou «colonne vertébrale » de ces figurations. (N otons au passage que la possibilité même de ces progressions mélodiques conjointes, voire de figurations, de figures répétées, est sans nul doute un élément de langage qui, à l'époque de la création de Treppenmusik, pouvait 
détonner par rapport à la disjonction et la non-répétition encore dominantes.) Le premier de ces remplissages est entendu dès le début, le trille do dièse - ré remplissant la quarte si-mi, le tout constituant un tétra corde symétrique $(2-1-2)^{5}$ (exemple 1) que l'on retrouve évidemment lors du retour de cette quarte à la fin de la section 19 (exemple 21). C'est sur ce tétracorde diatonique que s'articule la progression mélodique convergente des extrêmes lors de la contraction de l'accord muté de la section 2 (exemple 2, mes. 71 à 80 ). Les traits descendants de la section 4 (exemple 5 ) se déroulent également sur des gammes obtenues par la juxtaposition de trois de ces tétracordes. On observe aussi un autre remplissage de la quarte, chromatique celuilà, dans les sections $3 b$ (exemple 4 ) et 7 (exemple 8).

De plus, la quinte sera remplie par une structure intervallique de type 2-3-2 très proche de la gamme penta tonique. $0 \mathrm{n}$ trouve une amorce de ce remplissage lors des broderies opposées de l'accord de quintes superposées des mesures 86 à 97 (exemple 4). Dans la section 6 (exemple 7), les figurations pentatoniques illustrent également ce remplissage. $M$ a is on peut aussi voir ces dernières comme une horizontalité d'un accord de quintes superposées: la section 5 qui précède (exemple 6 ) nous y incite d'ailleurs, dont la formule mélodique répétée (si on ne considère que la partie supérieure) est clairement déduite d'un cycle de quintes partiel, qui constitue le mode pentatonique des figurations à venir. $\mathrm{N}$ ous touchons ici à la (très) ancienne idée des rapports entre le mélodique et l'harmonique, entre le vertical et l'horizontal, de même qu'aux théories qui veulent déduire les organisations modales (comme l'archétypal mode pentatonique) de cycles de quintes partiels. Un autre exemple frappant de ce rapport vertical/ horizontal est la section 8 où la mélodie est constituée purement et simplement du cycle de quintes, et où l'harmonie, articulée par des trilles, batteries lentes ou arpèges divers, est bâtie à partir d'extraits de ce même cycle (exemple 9). Ces harmonies, comme le mode pentatonique, nous font évidemment penser de nouveau à Debussy, mais le contexte et la syntaxe qui les organisent - cette succession en marche harmonique sans fin, le systématisme quasi mécanique de la mélodie des quintes - consacrent leur justification structurelle prop re, a u-delà de l'allusion stylistique. Cependant, l'articulation arpégée de ces harmonies et, quelques mesures plus tôt, le mouvement de la basse, cité au début de l'exemple 5 - depuis le si bémol, intégré à l'accord de quintes superposées, vers le la bémol puis le ré bémol, notes qui n'ont plus rien à voir avec cet accord - , ces articulations, donc, ce mouvement de basse et les accords qu'il crée ne sont pas là pour des raisons structurelles, à mon avis, mais plutôt pour l'évocation pure et simple, admirative et respectueuse, voire pour l'appropriation personnelle, l'absorption goulue, comme par mimétisme cannibale des sensualités du grand compositeur français... Ambiguitté de la «distance » postmoderne...
5. N otation conventionnelle des intervalles, où $0=$ unisson, 1 = seconde mineure, 2 = seconde majeure, 3 = tierce mineure, etc. Ici, 2-1-2 signifie, du grave vers l'aigu: seconde majeure - seconde mineure seconde majeure. 


\section{Cellules triphones}

La sortie de la citation du Sacre (section 12 b, exemples 15 et 16) donne lieu à l'installation d'un nouveau matériau (annoncé d'ailleurs par une petite cellule du saxophone ténor, très proche de la cellule initiale de l'«Introduction» du même Sacre) : il s'agit de cellules triphones - on est to ujours dans le conjoint.. - surtout diatoniques (2-2, parfois $1-2$ ou 2-1) mais quelquefois chromatiques, qui prennent le devant de la scène jusqu'à la fin de l'œuvre sous forme de figurations diverses: groupes rapides de quartes, quintes ou septièmes parallèles en doubles croches (section 13) (exemple 17); groupes plus lents, en noires, harmonisés en septièmes mineures parallèles, se déplaçant lentement vers le grave puis remontant, se décomposant en groupes triphones distincts de vitesses différentes superposées (noires, croches, triolets de croches, noires pointées) (section 14), bientôt exploités en trilles et batteries (section 15) qui se constituent eux-mêmes en trame de fond statique au-dessus de laquelle se dérouleront des lignes solistes aux instruments soprani (section 16) (exemple 18). Ce sont encore ces groupes de trois notes qui constituent le matériau des figurations de la «spirale ascendante sans fin » (section 17); ils se réduiront peu à peu à des groupes ascendants de deux notes, puis d'une note (!), se fondant en un trait ascendant, l'ascension de plus en plus rapide empêchant à la fin toute sous-articulation figurative. Plus loin, c'est la superposition de deux motifs triphones, l'un chromatique et l'autre diatonique, qui engendrera les accords exploités dans les sections 18 et 20 (exemple 19). Enfin, la section 19 présente elle aussi une lente progression ascendante d'un cluster diatonique triphone sur un mode dont on a souligné plus haut la structure (2-1-2-2-1-2-, etc.) (exemple 20). En bout d'ascension, l'inscription de ce cluster dans la quarte si-mi et sa mutation en trille do dièse - ré (exemple 21 ) ramènera le matériau initial de l'œuvre.

Encore une fois, l'héritage de Debussy et de Stravinski plane au-dessus de ces cellules triphones et de leur articulation en figures répétées. C es compositeurs ont en effet développé des techniques audacieuses de déduction sur le plan mélodico-harmonique en travaillant à partir de microcellules. Mais Rea transmuera cet héritage en le croisant avec celui de Steve Reich et des modernes.

\section{Série harmonique}

Se détachant des harmonies déduites selon les logiques intervalliques que nous venons d'exa miner, les quatre accords de la section 9 , composés des douze premiers partiels de la série des harmoniques naturels (déflexions non tempérées 
comprises, pour les septième et onzième harmoniques), gardent cependant cela en commun a vec les matériaux précédents qu'ils se succèdent en montant chaque fois d'une quarte juste (fondamentales: mi, la, ré, sol). Ils produisent bien sûr un très agréable (et très euphonique) contraste, mais également un autre renvoi à un archétype musical bien connu. Le premier de ces quatre accords apparaît à l'exemple 12.

\section{Espace-temps (géométrie, formes, illusions)}

\section{Autres engendrements, mutations et transitions harmoniques}

$\mathrm{N}$ ous sortirons maintenant de l'«hors temps» pour observer quelques manipulations qui se déroulent dans le temps et affectent les éléments des familles harmoniques précitées. Ces manipulations engendrent de nouvelles harmonies plus passagères ou encore donnent lieu à des mutations d'agrégats ou à des transitions entre ceux-ci. C'est aussi à ce niveau syntaxique d'unités formelles intermédiaires que s'accomplira une partie du travail de recontextualisation des harmonies codées dont nous venons de faire un survol.

Le procédé bien connu de la transposition est utilisé dans la section 4 où les mesures 121-122 et 123-124 reproduisent ce qui a été entendu dans les mesures 118 à 120 (exemple 5). Le rôle de cette transposition pourrait être de mener progressivement les pôles supérieurs de la grille statique de quartes vers le do dièse, sommet de la formule mélodique en quintes parallèles de la section suivante. Ce qui était fixé et répétitif dévie à la fin, cherche à s'inscrire dans un devenir, dans une forme plus large.

II faut mentionner ensuite la mutation de l'accord de quartes superposées, obtenue en remplaçant la quarte centrale par une quinte (exemple 2 ), d'où division en deux accords de cinq quartes superposées et écartement symétrique de ceuxci. Cette mutation se produit au moment où l'expansion des mesures 65 à 70 aurait pu atteindre l'accord dodécaphonique (accord comprenant le cycle complet des quartes). La surprise que cause cet aboutissement inattendu - bien loin de la résolution qu'en aura it conçu Schönberg - se trouve amplifiée par un effet de contraste, un pianissimo subito que des traînées de réinjection électracoustique recouvrent momentanément.

On a déjà signalé le processus de contraction de ce même accord muté, un processus accompli par la progression mélodique convergente des notes extrê- 
mes (exemple 2). Un autre type de contraction, également élaborée à partir de l'accord de quartes, est entendu pendant la section $3 \mathrm{~b}$. Les accords qui se trouvent aux sommets de mouvements d'expansion-contraction se déroulant sur deux mesures font eux-mêmes partie d'un processus de contraction, mais la mutation est plus prononcée cette fois-ci puisqu'elle affecte la structure même de l'accord (exemple 4). 0 n notera, d'étape en étape, les structures intervalliques symétriques de ces accords-sommets autour d'une note centrale, soit, du grave à l'aigu, les intervalles suivants: $5555555555,4545445454,4444334444,34342$ $24343,3333113333,23233232,22211222$. O n remarquera aussi l'alternance de la note centrale entre le si ou le do, ce qui permetl'utilisation de deux transpositions différentes d'une même structure intervallique pour l'expansion et la contraction de l'accord. L'effet de cette contraction des accords-sommets est d'autant plus saisissant qu'une accélération l'accompagne: contraction simultanée de l'espace et du temps, mais paradoxalement, et a vec subtilité, Rea allongera peu à peu le creux des mouvements de vague, empêchant l'accélération de se produire trop rapidement Peutêtre est-ce pour conserver tout son impact à l'autre grand mouvement d'accélération que constitue la «spirale» de la section 17. L'originalité du processus total absorbe, du moins dans cet exemple, les quelques éléments qui pourraient évoquer un quelconque passé : la métrique, la périodicité du mouvement d'expansion-contraction, les fusées chromatiques.

La mutation intervallique symétrique affecte également les accords parallèles qui harmonisent les groupes triphones en doubles croches (section 13) (exemple 17): 5-4-5 devenant 7-2-7 puis 10-0-10. Si les voix extrêmes évoluent en expansion, les voix internes se rapprochent et fusionnent (laissant d'ailleurs libre, par le fait même, un groupe de trois instruments), le tout créant l'effet d'une "modulation» vers la gamme par tons sur laquelle va se dérouler la section suivante. La pensée géométrique intègre, ici aussi, le pouvoir allusif des matériaux mis en jeu.

Une autre mutation intervallique très intéressante est celle qui procède par transposition a symétrique des deux intervalles extrêmes d'un accord initial de quatre sons (7-7-2) (section 18) (exemple 19): la seconde supérieure est transposée par ton lorsque située au-dessus de l'axe de départ, par demi-tons lorsque pla cée au-dessous, et vice versa pour la quinte inférieure. Comme on l'a déjà mentionné, les mouvements triphones chromatique et diatonique contribuent ici à la création d'entités harmoniques nouvelles, disposées de façon symétrique autour d'un centre (du grave à l'aigu: 7-9-2, 7-8-2, 7-7-2, 7-8-2, 7-9-2). L'accord de départ (7-7-2) était lui-même un hybride curieux, issu d'un accord défectif de quintes superposées sol-ré-la-(mi)-(si)-fa dièse sautant d'une quinte vers ré-la-mi-(si)-fa dièse (début de l'exemple 18). C hacun de ces accords pris individuellement pourrait éveiller des souvenirs to naux ou modaux, mais leur superposition et entremêlement aux tro is familles instrumentales dans les sections 18 et 20 brouillent habilement toute évocation fonctionnelle au profit d'un travail attentif des couleurs. L'accord final (exemple 22 ) résulte d'ailleurs d'une superposition de deux transpositions 
différentes de l'un d'entre eux (7-8-2), à distance de quarte (sur les basses si et mi entendues au début de cette section terminale, comme au début de l'œuvre !): accord suspensif, quasi-total diatonique. II serait soutenu ou répété qu'on l'entendrait davantage comme l'un de ces accords consonants non résolus dont Steve Reich colore ses longues plages de temps (dans 0 ctet, par exemple). Mais ici le continuum s'interrompt, comme sur trois points de suspension.

Les transitions encadrant la section 9 (accords de séries harmoniques) valent aussi la peine d'être étudiées. Alors que la transition d'entrée, par le moyen d'un flou très bref, opérait une mutation rapide depuis le pentatonisme des quintes qui précédaient, la transition de sortie est plus élaborée. Elle superpose plusieurs types de déplacements intervalliques (exemple 13) - déplacements chromatiques ou dia toniques plus ou moins convergents dans les basses, parallélisme descendant d'accords majeurs aux ténors, expansion puis recontraction intervallique chez les altos $(22,32,33,23,22)$, statisme sur un ensemble triphone diatonique chez les soprani - chacune de ces «voix» préparant l'espacement de septièmes mineures et le contexte de gamme par tons qui suivront. $M$ ais avant que cette stabilisation ne se produise, l'espace harmonique aura subi une véritable torsion. Je pense à cette sphère dessinée par Escher qui réfléchit de façon courbée le décor qui l'entoure. Cette transition force l'admiration: sortir d'une zone harmonique où la gravitation, la polarisation est aussi prononcée était en soi un défi. Les compositeurs spectraux en savent quelque chose, qui ont aussi joué avec ce problème.

L'évolution des trois éléments triphones diatoniques superposés au cours des sections $15 b$ et 16 est aussi remarquable (exemple 18). Elle se déroule dans un médium grave bourbeux et dans un contexte de brouillage par superposition rythmique. Les triphones externes se déplacent symétriquement (à la quinte, à la sixte majeure, à la septième mineure, à la quarte) autour du triphone central fixe (doré-mi), en créant des ensembles harmoniques parfois proches du total dia tonique ou de la gamme par tons et parfois plus dissonants.

Le long exposé qui précède voulait mettre en évidence les relations qui existent entre les différentes familles d'éléments sur le plan harmonique et faire ressortir l'esprit d'exploration quasi géométrique qui a inspiré l'invention des techniques d'élaboration et de déduction sur ce même plan. De temps à autre, j'ai aussi voulu souligner que cette manipulation sophistiquée mais cependant très claire d'objets familiers mène à une recontextualisation de ceux-ci, au-delà de toute nostalgie.

Les mêmes remarques pourraient s'appliquer, si l'on s'attardait à l'articulation de ces entités harmoniques, au comportement motivique, à l'organisation des figurations. $0 \mathrm{n}$ trouverait sans peine les progressions numériques qui ordonnent les sections $1,2 a, 2 b, 9 a$, de même que les ponctuations de la section 19. Le parallélisme, les canons, l'imitation, la continuité rythmique, la référence à une pulsation, à une métrique constantes sont des techniques et des faits musicaux «vieux comme le monde », mais ils semblent qu'ils n'avaient pas encore dit tout ce qu'ils 
avaient à dire..., tout étant, encore une fois, dans la manière et dans l'esprit de cette manière.

Par exemple, même si l'on pense à Steve Reich en entendant certaines des harmonies consonantes et en remarquant des procédés qui conjuguent répétition et transformation graduelle, la différence stylistique est patente. II ne s'agit pas d'un processus systématique élaboré sur une longue période, grâce à un rythme de changement plutôt lent qui donne l'illusion momentanée du statisme. II ne s'agit pas non plus d'un «motivisme » à figurations, à ostinati et superposition comme dans le Sacre, ni d'un thématisme classique. La fonctionnalité classique, la «directionnalité » tonale, définie par les cadences et les plans de tonalités, a aussi été remplacée par une autre fonctionnalité, par d'autres types de directionnalités définies à même les procédés «manipula toires» employés.

Carpas une section de Treppenmusik ne peut vraiment être considérée comme statique ou non directionnelle. Aux directionnalités déjà mentionnées, et qui touchent le plan harmonique, s'ajoutent celles des articulations figurationnelles et des microstructures. Parcourons l'œ uvre de nouveau selon cette perspective : section 1) pour chacune des cinq sous-sections, accelerandos de la succession des trilles; 2 ) expansion puis contraction des quartes, ascension puis descente des quintes parallèles; 3 ) développement par contraction spatiale et temporelle; 4) transposition ascendante de l'enchevêtrement des traits descendants vers la fin de la section; 5 ) mutation timbrale de la mélodie répétée en quintes parallèles; 6) sur les figurations pentatoniques, ouverture depuis l'aigu puis fermeture vers le grave d'un filtre timbro-registral; 7 ) mutation timb ro-reg istrale des descentes chromatiques partiellement trillées; 8) forme en arche créée par l'augmentation de la longueur des cellules mélodiques de quintes, suivie de leur diminution (3 croches - 5 - 7 - $5-3$ ); 9) ascension par quartes de l'accord de série harmonique et mutation pour sortir de ce type d'harmonie; 10 et 11 ) descente puis remontée; 12) mutation harmonique pour la sortie de la citation de Stravinski; 13) expansion harmonique des cellules triphones en doubles croches; 14 ) descente puis remontée des cellules triphones en tuilage en noires et établissement graduel d'une superposition de vitesses orientée vers un ralentissement (doubles croches, triolets de croches, croches, noire pointée); 15 ) épaississement harmonique par superposition de cellules triphones, puis mutation harmonique; 16 ) malgré un fond relativement statique, perception d'une longue ligne mélodique soliste aux soprani et forme en arche des tierces successives (exemple 18); 17) malgré le côté «sans fin» de la spirale, accélération inexorable; 18 ) succession harmonique articulée en hoquet, puis en trilles, puis en mélange trilles/ notes répétées (donc : granulation sonore croissante), puis réduction orchestrale et diminuendo-disparition dans la rétroaction; 19) ascension de la cellule triphone combinée à un accelerando de ponctuations staccato aux ténors, puis mutation du remplissage de la quarte si-mi, accomplie selon un flou habile; 20) coda, peutêtre la plus statique des sections, tournant sur ellemême, étrange pulsation de couleurs bruta lement interrompue. 
Cette liste confirme le dynamisme formel que nous percevons à l'audition de Treppenmusik: pas une section qui ne nous mène quelque part $Q$ uant à la forme générale, on perçoit des directions à plus grande échelle ou des regroupements de plus grandes unités, par exemple sur le plan harmonique:

- dominance des quartes: sections $1,2 a$ et $b, 3,4$ (en septièmes) et 7 (les quartes y sont cependant plus virtuelles à cause du remplissage chromatique);

- dominance des quintes: sections $2 c$ et d (annonce des quintes), 5,6 et 8 ;

- puis, après l'accord de série harmonique (section 9), dominance de la gamme partons (sections 10 et 11 , puis, plus tard, sections 14 et 17);

- après la citation de Stravinski, dominance des motifs triphones (induits par Stra vinski) et des harmonies engendrées par eux: sections 13 à 19 ;

- enfin, dominance terminale des accords asymétriques spéciaux: sections 18 à 20 .

O n pourrait aussi chercher et trouver d'autres directions formelles globales ou d'autres regroupements de sections en considérant, par exemple, les types de textures et de figurations; ou encore en considérant les zones «registrales»- statismes ou mouvements généra ux vers le grave ou l'aigu; ou encore les tempi zone de stabilité et de mouvements directionnels; etc. Un schéma global qui prendrait en charge plusieurs paramètres fera it ressortir - on peut en être sûr l'importance formelle de la section 17 , celle que j'ai appelé la "spirale sans fin ${ }^{6} »$. Un défi pour les interprètes, un point focal pour l'auditeur, cette section méritera it plusieurs pages à elle seule pour en souligner l'habileté de construction.

Le périple formel de l'œuvre comporte aussi des ajouts et des transitions (dont certaines ont déjà été mentionnées) qui changent la succession des processus en une suite organique et la criblent de surprises. Ainsi, le clin d'œil à Debussy, qui sert d'introduction à la section 3 (mes. 86 à 87 ); le tuilage des sections 4 et 5 ; les basses des mesures 130 à 132, qui annoncent les matériaux triphones de même que les ascensions des accords de quintes de la section suivante; les soudains glissandi de cordes qui préparent la section 7 ; le solo des soprani de la section 16 ; la clausule terminant la spirale de la section 17 ; le point d'orgue sur un accord d'harmoniques de cordes trémolo se fondant dans la rétroaction électroacoustique (fin de la section 18), ainsi que le cri aigu et la rapide descente qui y met bruta lement fin. Les transformations électroa coustiques participent autant aux mécanismes qui fabriquent les illusions et les ombres qu'à cette souplesse d'invention qui fait respirer l'œ uvre.

Les directionna lités mentionnées plus haut contredira ientelles ce que l'on aurait pu attendre en consultant la référence aux boucles et escaliers sans fin d'Escher dans la notice citée de Rea? Est-il vraiment possible de suggérer une boucle de
6. Dans les notes à la fin de la partition de son œuvre pour piano Las M eninas, John Rea explique que les «roulades perpétuellement ascendantes » de cette section de Treppenmusik sont un «commentaire musical » du phénomène électroacoustique connu sous le nom de "sons de Shepard». II mentionne que ce phénomène est aussi décrit dans le livre de Douglas $R$. Hofstadter, G ödel, Escher, Bach (1979, p. 718 ), en relation avec le canon sans fin de l'O ffrande musicale de Bach et les escaliers impossibles de Escher. Ces sons paradoxaux ont également été développés par le compositeur Jean-C laude Risset dans certaines de ses œuvres électroacoustiques à partir de 1968. Les longues descentes sans fin de la «Région IV » des Hymnen de Karlheinz Stockhausen relèvent d'une technique semblable. 
Temps en musique ? Et pourtant, tout ce périple de montées et de descentes, d'accélérations et de ralentissements, d'expansions et de contractions, ne nous a-t-il pas ramenés à notre point de départ: à la quarte et à son cluster diatonique?

D'une façon générale, je dirais qu'avec Treppenmusik, John Rea a proposé une lecture dramatique de l'œ uvre de l'artiste néerlandais. M usique conçue hors plan pour saisir l'instant présent et la nécessité suivante, musique construite attentivement comme une mécanique céleste, elle nous mène, comme promis, à la fois quelque part et nulle part.

L'analyse complète, qui saisit to ut, n'existe évidemment pas. Il y aurait tant d'autres détails à relever, tant d'autres perspectives à adopter. Ainsi, je n'en étais ici qu'au début d'une vision synthétique sur le plan de la forme. On dit souvent que les grandes œuvres révèlent sans cesse de nouvelles facettes d'elles-mêmes aux générations d'auditeurs qui se suivent. Si Treppenmusik est pour vous l'une de celleslà, c'est votre tour de fouiller a fin d'éclairer et peutêtre d'accroître votre plaisir.

\section{Conclusion}

Dix-huit ans après la création de Treppenmusik, vingt-sept ans après celle de Chants de Claude Vivier (1973), qui révéla sa voie au jeune compositeur, vingt-six ans après le début du cycle des Espaces acoustiques de $\mathrm{G}$ érard $\mathrm{G}$ risey (1974), pour ne pas mentionner les œuvres mutantes de compositeurs de la génération précédente (Votre Faust et $C$ ouleurs croisées d'H enri Pousseur, $M$ a ntra de Stockha usen, etc. ), a près tout un corpus d'œuvres qui se sont réclamées du postmodernisme musical ou qui peuvent s'y apparenter, ce courant estil toujours d'actualité (si tant est qu'il existe... ) ou est-il devenu un nouvel académisme? Sa nécessité dans les années 1970-1990 ne faisait pas de doute. Maintenant, il fait l'objet de publications synthétiques ${ }^{7}$. II côtoie les autres esthétiques/ attitudes dans un éclectisme que d'aucuns décrient. Surtout lorsque des musiques à "sonorité sérielle ", qu'il était devenu de bon ton de condamner (Boulez, Berio, Ferneyhough et une flopée de compositeurs de la plus jeune génération qui restent près de ce langage ou qui le croisent avec les acquis de l'école spectrale), atteignent à un assouplissement et à un accomplissement expressif remarquable (ce constat vaudrait aussi pour les compositeurs de l'école spectrale).

Q uand la ressemblance entre les musiques de plusieurs compositeurs devient gênante, on peut commencer à craindre l'académisme, et ceci peut toucher tous les courants musicaux. II ne sert plus à rien de jeter des a nathèmes stylistiques, on le sait, mais cependant, demeure toujours l'exigence de découvrir sa propre voie et de la défricher plus avant.
7. Comme cet excellent M usique et postmodernité de Béatrice Ramaut-C hevassus (1998). Le lecteur pourra également se référer à la note 4 de l'article de Friedemann Sallis dans ce numéro. (N .D.É.) 
Sur un autre plan, à l'absurde, s'il n'éta it que référentiel, le postmodernisme serait peutêtre pur collage. Je ne doute pas qu'il pourrait atteindre malgré tout à une sophistication et à une densité remarquables, voire donner naissance à un style, alors même qu'on assisterait à une espèce d'appauvrissement par dépersonnalisation sur le plan du contenu musical. Le postmodernisme est peutêtre plus intéressant s'il joue avec des allusions et s'il garde une certaine distance avec ses affections, s'il demeure critique. Ce qui ne signifie pas ne pas céder! II s'agit de céder avec organisation... car c'est de l'Art C'est, il me semble, à ce postmodernisme-là auquel appartiendra it John Rea.

Le postmodernisme participe à sa manière au renouvellement du langage. $0 \mathrm{n}$ pourrait le voir comme le courant qui comble les trous évolutifs, qui peuple les terres intermédiaires traversées trop rapidement et négligées par les a vant-gardes. Et qui, pour renouer avec la citation de Pousseur, cherche à comprendre la signification de ces espaces plus proches, plus familiers, mieux: à leur donner un nouveau sens, c'est-à-dire, en fait, à inventer d'autres plaisirs...

G O N N EVIUE, M. (1990), «Humeurs postmodernes», C ircuit, vol. I, n 1, p. 49-61.

HO FSTADTER, Douglas R. (1979), Gödel, Escher, Bach, N ew York, Vintage Books.

PO USSEUR, H. (1968), "L'apothéose de Rameau. Essai sur la question harmonique », Revue d'esthétique, $n^{0} 21$, «Les musiques nouvelles», Paris, Klincksieck, p. 105-172.

RAM AUT-C HEVASSUS, B. (1998), M usique et postmodernité, Paris, Presses universitaires de France, coll. "Q ue sais-je ?», $n^{0} 3378,128$ p.

REA, J. (1991), Las M eninas, partition, M ontréal, M usigraphe, 52 p.

REA, J. (1982), Treppenmusik, C entre de musique canadienne au Q uébec.

RO SEN , C . (1993), Plaisir de jouer, plaisir de penser. Entretiens avec C atherine Temerson, Paris, éditions Eshel, 82 p. 


\section{AN NEXE: Tableau de la forme générale}

À titre de référence, le tableau suivant illustre brièvement la forme de Treppenmusik. Les sections sont définies par des textures, des figurations ou des développements «processuels » distincts.

\begin{tabular}{|c|c|c|c|}
\hline Section & Mes. & Description brève & Ex. \\
\hline 1 & 1 & Introduction en 5 sous-sections: cluster diatonique de $4^{\text {te }}$. & 1 \\
\hline $2 a$ & 65 & $\begin{array}{l}\text { Expansion vers un accord dodécaphonique de } 4^{\text {tes }} \\
\text { superposées. }\end{array}$ & 2 \\
\hline $2 b$ & 71 & $\begin{array}{l}\text { Mutation de l'accord de } 4^{\text {tes }} \text { ( } 5^{\text {te }} \text { au centre) et } \\
\text { contraction par convergences des extrêmes. }\end{array}$ & 2 \\
\hline $2 c$ & 81 & $\begin{array}{l}\text { Ascension chromatique d'un accord de trois } 5^{\text {tes }} \\
\text { superposées. }\end{array}$ & 3 \\
\hline $2 d$ & 84 & $\begin{array}{l}\text { Descente du même accord avec déformation. - } \\
\text { Point d'orgue. }\end{array}$ & 3 \\
\hline $3 a$ & 86 & Broderie partielle du même accord (intro. de $3 \mathrm{~b}$ ). & 4 \\
\hline $3 b$ & 98 & $\begin{array}{l}7 \text { mouvements d'expansion-contraction, les } \\
\text { accords-sommets de ceux-ci eux-mêmes en contraction. }\end{array}$ & 4 \\
\hline 4 & 117 & Descentes en gammes sur grille de $7^{\text {mes }}$. & 5 \\
\hline 5 & 125 & $\begin{array}{l}\text { Réitération d'une mélodie penta tonique de } 8 \text { notes, } \\
\text { harmonisée en } 5^{\text {tes }} \text { parallèles. }\end{array}$ & 6 \\
\hline 6 & 134 & Arpèges penta toniques, rapides et lents. & 7 \\
\hline 7 & 139 & $\begin{array}{l}\text { G rille de } 4^{\text {tes }} \text { remplie par des descentes chromatiques } \\
\text { tillées. }\end{array}$ & 8 \\
\hline $8 a$ & 165 & $\begin{array}{l}5 \text { cycles de } 5^{\text {tes }} \text { exposés mélodiquement sur fond } \\
\text { alternativement penta tonique et hexa tonique. }\end{array}$ & $\begin{array}{l}9,10 \\
11\end{array}$ \\
\hline $8 b$ & 205 & Transition. & \\
\hline $9 a$ & 213 & $\begin{array}{l}\text { Accord de série harmonique: } 4 \text { fondamentales } \\
\text { successives. }\end{array}$ & 12 \\
\hline $9 b$ & 225 & Mutation/ préparation. & 13 \\
\hline 10 & 253 & Descente, grille de $7^{\text {mes }}$, gamme par tons. & 14 \\
\hline 11 & 262 & Remontée en gamme par tons. & \\
\hline
\end{tabular}




\begin{tabular}{|c|c|c|c|}
\hline $12 a$ & 282 & Citation Stravinski. & 15 \\
\hline $12 b$ & 293 & Sortie de citation, début des cellules triphones. & 16 \\
\hline 13 & 305 & $\begin{array}{l}\text { Cellules triphones en doubles croches, alternance } \\
\text { des familles instrumentales. }\end{array}$ & 17 \\
\hline 14 & 317 & $\begin{array}{l}\text { Descente puis remontée, cellules triphones sur gamme } \\
\text { par tons, ralentissement. }\end{array}$ & \\
\hline $15 a$ & 338 & Épa ississement harmonique par ajout de cellules triphones. & \\
\hline $15 b$ & 354 & Mutation harmonique. & 18 \\
\hline 16 & 354 & Solo des soprani sur fond harmonique statique. & 18 \\
\hline $7 a$ & 387 & Spirale ascendante et accelerando sans fin. & \\
\hline $17 \mathrm{~b}$ & 428 & Clausule de la spirale. & \\
\hline $18 a$ & 437 & Exposition des accords asymétriques en hoquet. & 19 \\
\hline $18 b$ & 442 & $\begin{array}{l}\text { Les mêmes, trillés ou en notes répétées. } 0 \text { mbre... } \\
\text { Point d'orgue. }\end{array}$ & \\
\hline 19 & 460 & $\begin{array}{l}\text { «Cri aigu» et descente rapide. M ontée en tuilage } \\
\text { de cellule triphone. M utation du remplissage de la } \\
4^{\text {te }} \text { si-mi, retour du cluster diatonique initial. }\end{array}$ & $\begin{array}{l}20 \\
21 \\
22\end{array}$ \\
\hline 20 & 487 & $\begin{array}{l}\text { Coda sur accords asymétriques trillés ou en notes } \\
\text { répétées. } 3 \text { ponctuations finales. }\end{array}$ & 19 \\
\hline
\end{tabular}

Dans les exemples musicaux, les numéros de sections sont encadrés.

\section{John Rea}

\section{Notice biographique}

John Rea (1944) mène une triple carrière de compositeur, de professeur et d'animateur. ॥ a étudié la composition à l'U niversité de Toronto (sa ville natale) et à l'Université Princeton. Parmi ses récentes créations, mentionnons: un septuor, Plus que la plus que lente pour L'Ex Novo Ensemble (créé à Venise en 1998); une réorchestration pour 21 musiciens de l'opéra W ozzeck d'Alban Berg, pour le $\mathrm{N}$ ouvel Ensemble Moderne (créée à Banff et à Montréal en 1995). Depuis 1973, il enseigne la composition et la théorie musicale à la Faculté de musique de l'Université McGill, dont il a été le doyen de 1986 à 1991.

\section{John Rea}

\section{Liste sélective des œuvres}

Les Jours, 1968-1969 (30 min). Ballet pour grand orchestre. Éditions C entre de musique canadienne. 
0 ver Time, 1987 (11 min). 0 rchestre [2-2-2-2 / 4-2-3-1 / perc / cordes]. Éditions Centre de musique canadienne.

Time and Again, 1987 (12 min). 0 rchestre [2-2-2-2 / 2-2-0-0 / 2 perc / pno / cordes]. Éditions $C$ entre de musique canadienne.

Las M eninas, 1990-1991 (30 min). Piano ( 21 variations «transformelles» sur les Kinderszenen de Robert Schumann). Éditions $C$ entre de musique canadienne.

0 bjets Perdus, 1991 (18 min). Q uatuor à cordes. Éditions $C$ entre de musique canadienne.

\section{John Rea}

Discographie sélective

Treppenmusik, Ensemble de la SMCQ, dir. Serge G arant Centredisques, CM C 205 , 1986, C M C -CD 5194, «M ontréal Postmoderne».

0 ver Time, 0 rchestre M étropolita in, dir. W alter Boudreau. Centredisques, C M C-C D 3188,1988 , 0 rchestre Métropolitain ».

«Pulau Dewata » (orchestration) de Claude Vivier, N ouvel Ensemble Moderne, dir. Lorraine Vaillancourt UM M US, UM M 104, 1992, «Bali à M ontréal».

0 ffenes Lied, Ensemble de la SM C Q, M arie-Danielle Parent et Yolande Parent, sopranos, dir. W alter Boudreau. Doberman-Yppan / Radio-C anada, DO 135, 1992, «SMCQ». 


\section{Treppenmusik de John Rea Analyse de Michel Gonneville \\ Exemples musicaux}
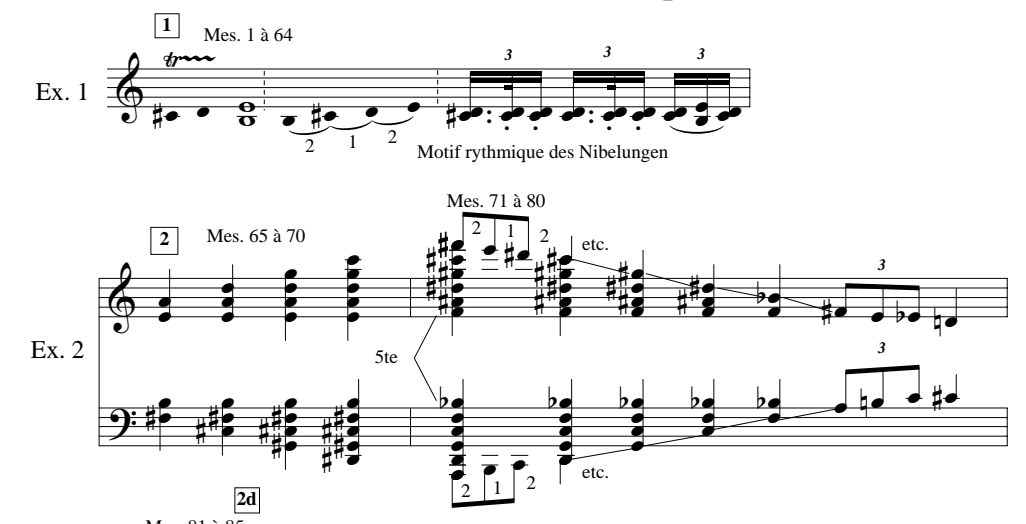

Ex. 3
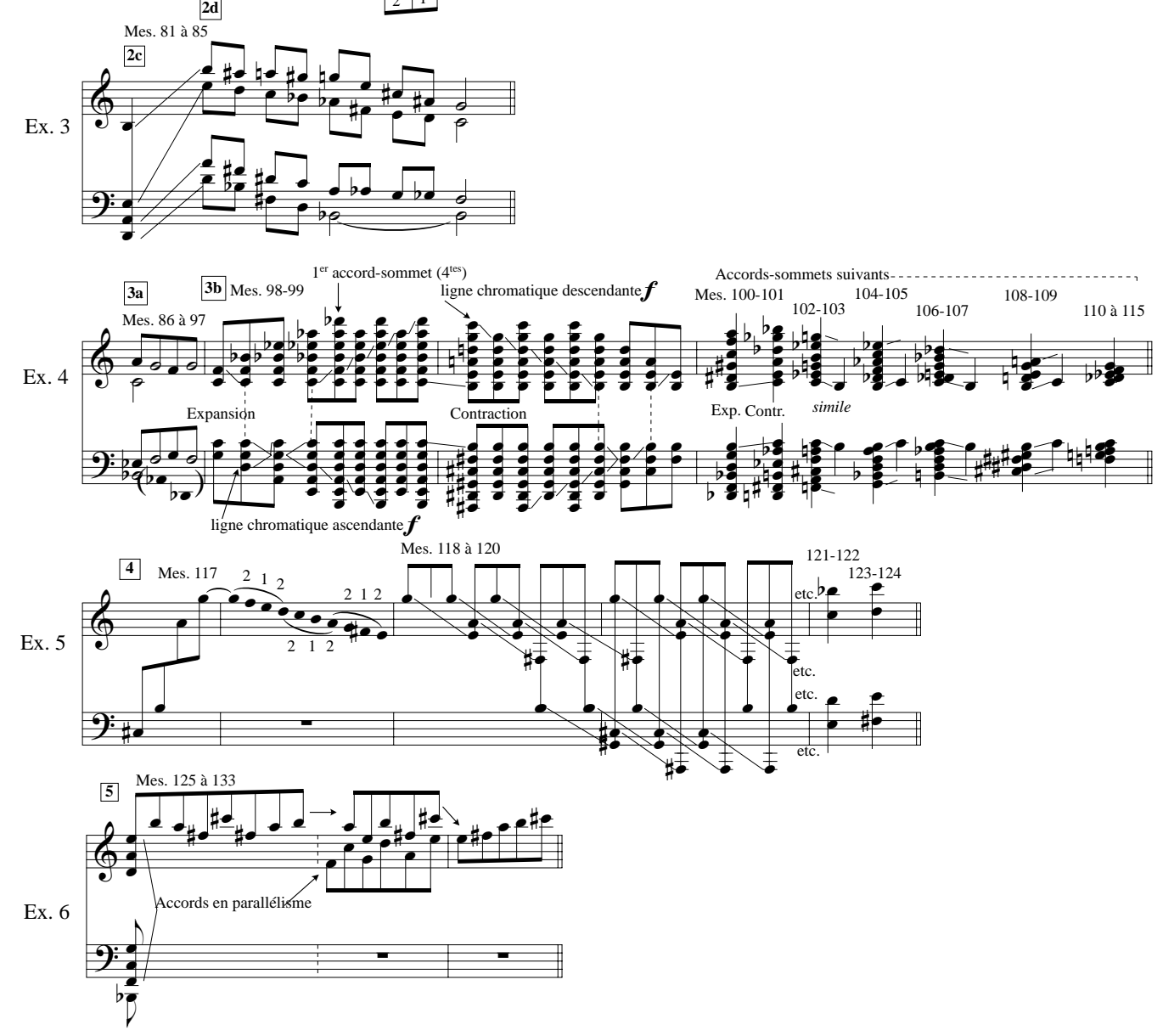
Ex. 7
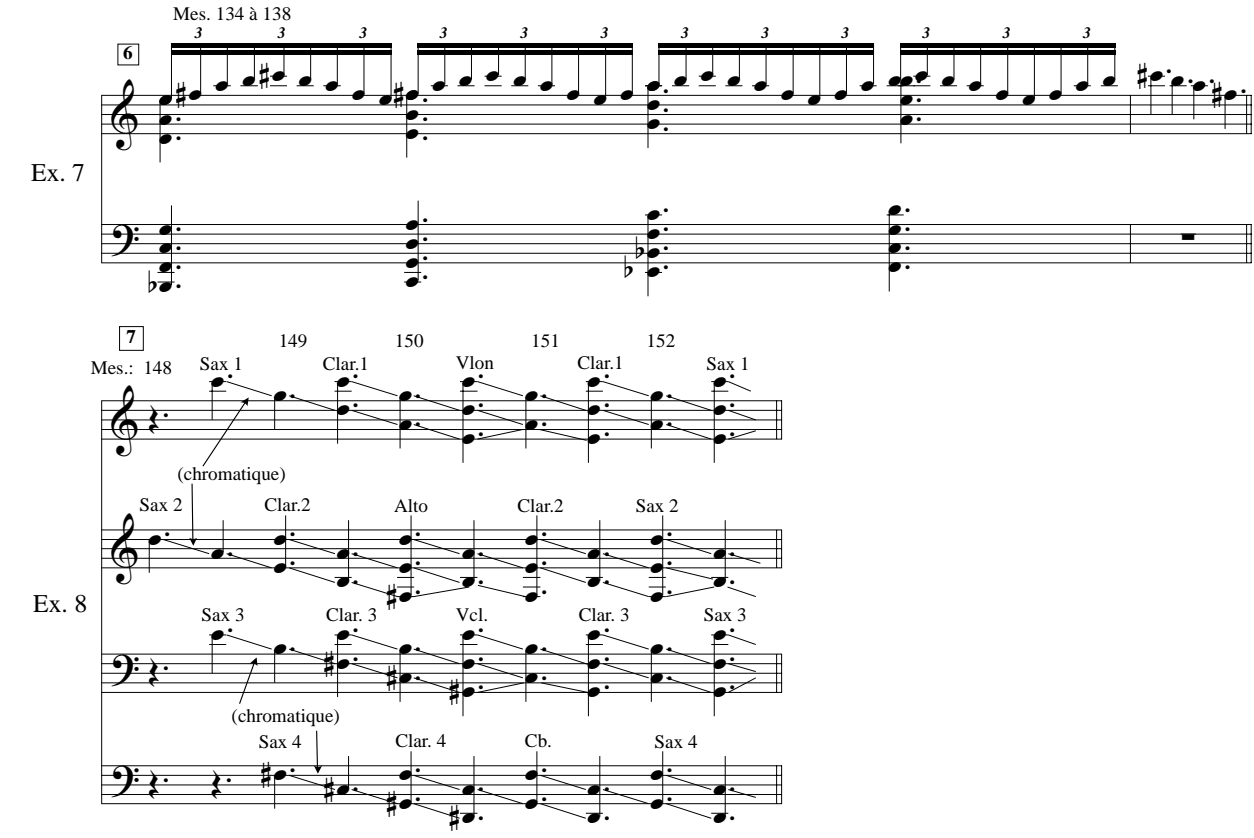

8 Mes. 165 à 169 Cellules de 3 croches.

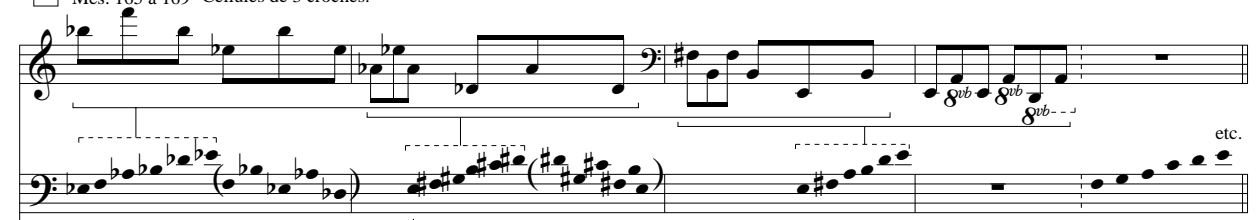

Ex. 9

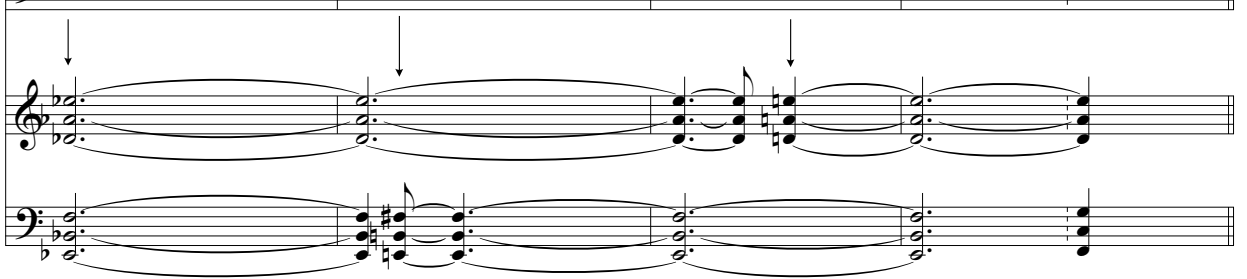

Mes. 178 à $187 \quad$ Cellules de 7 croches.

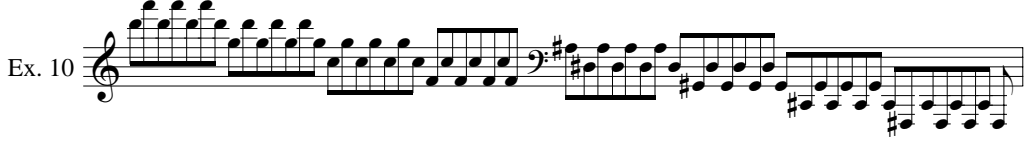

Mes. 191 à 197 Cellules de 5 croches.

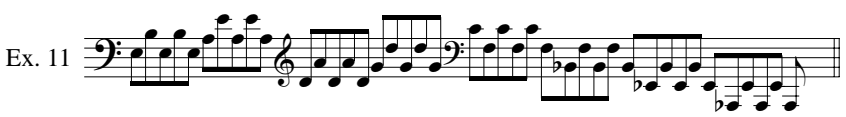



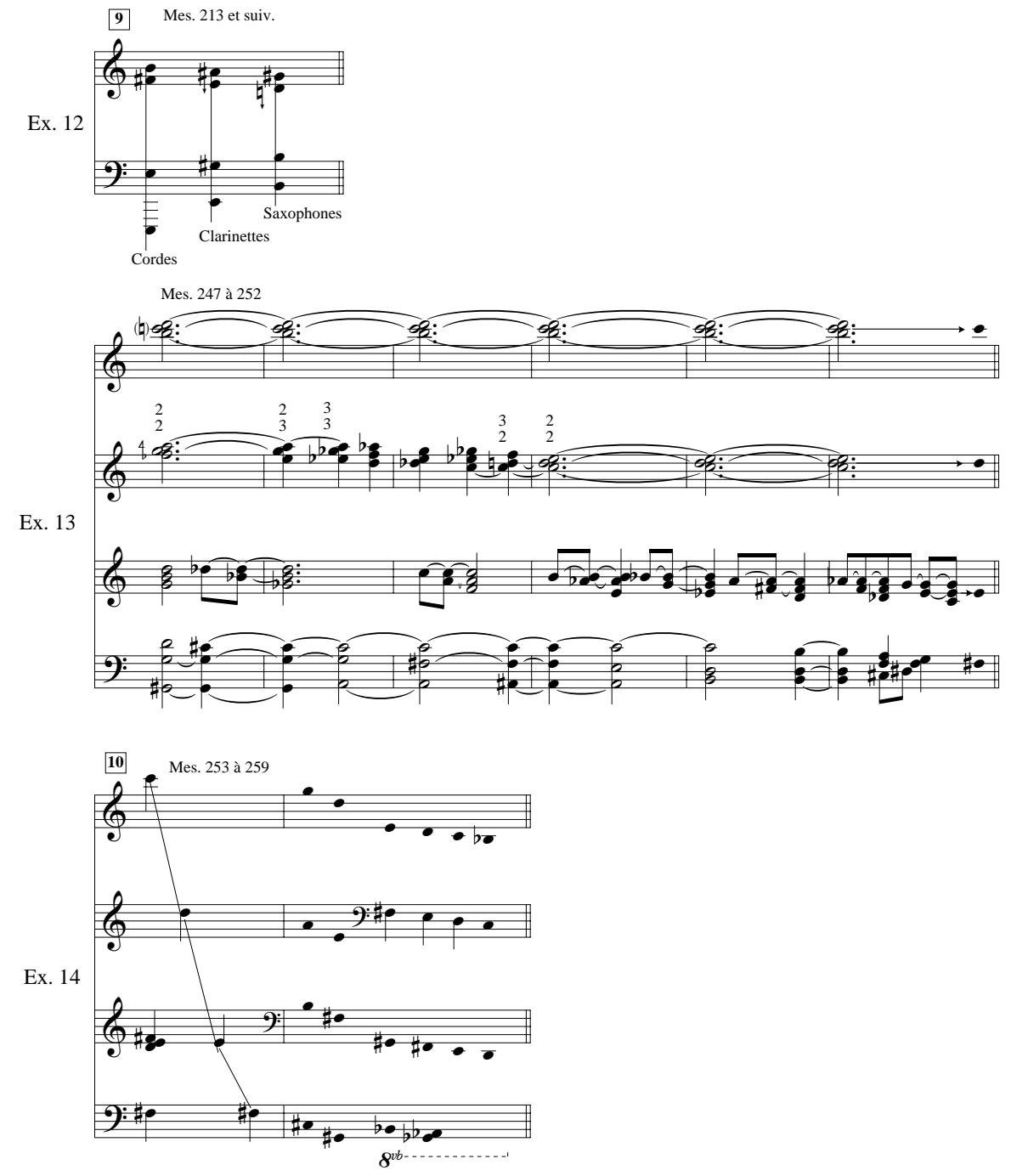
Ex. 15

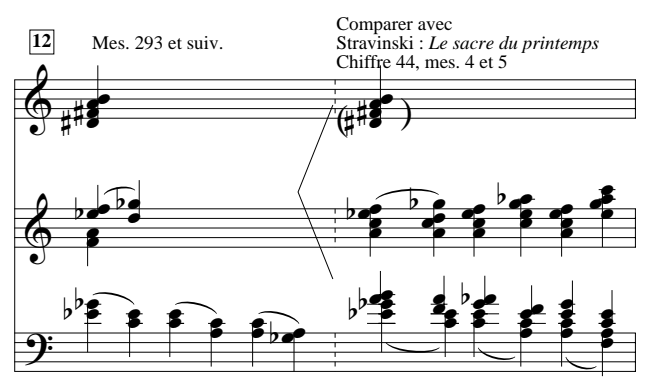

Ex. 16

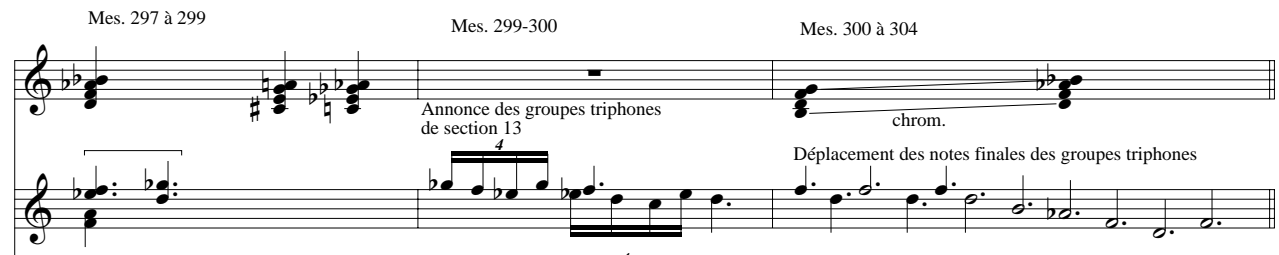
Cellule triphone diatonique

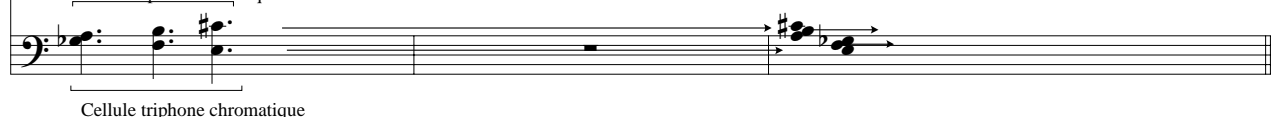

Cellule triphone chromatique

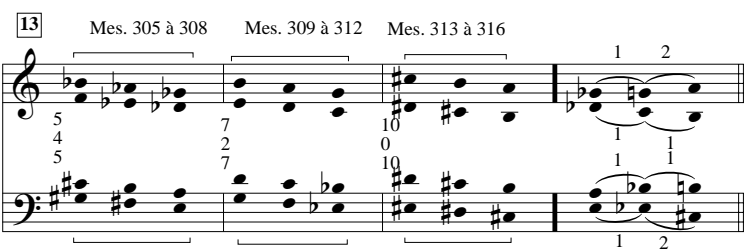

Ex. 18

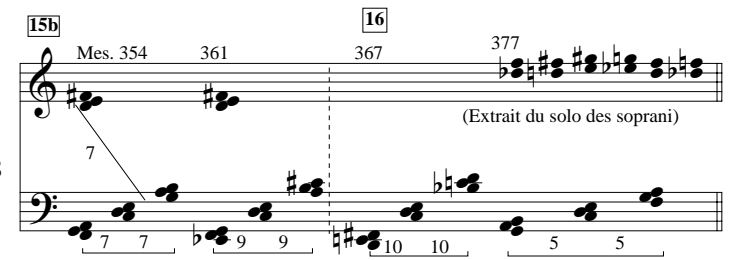

Ex. 19

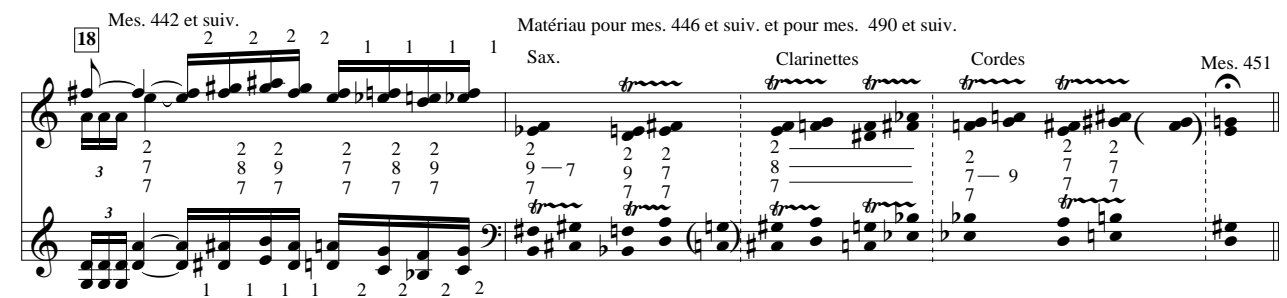



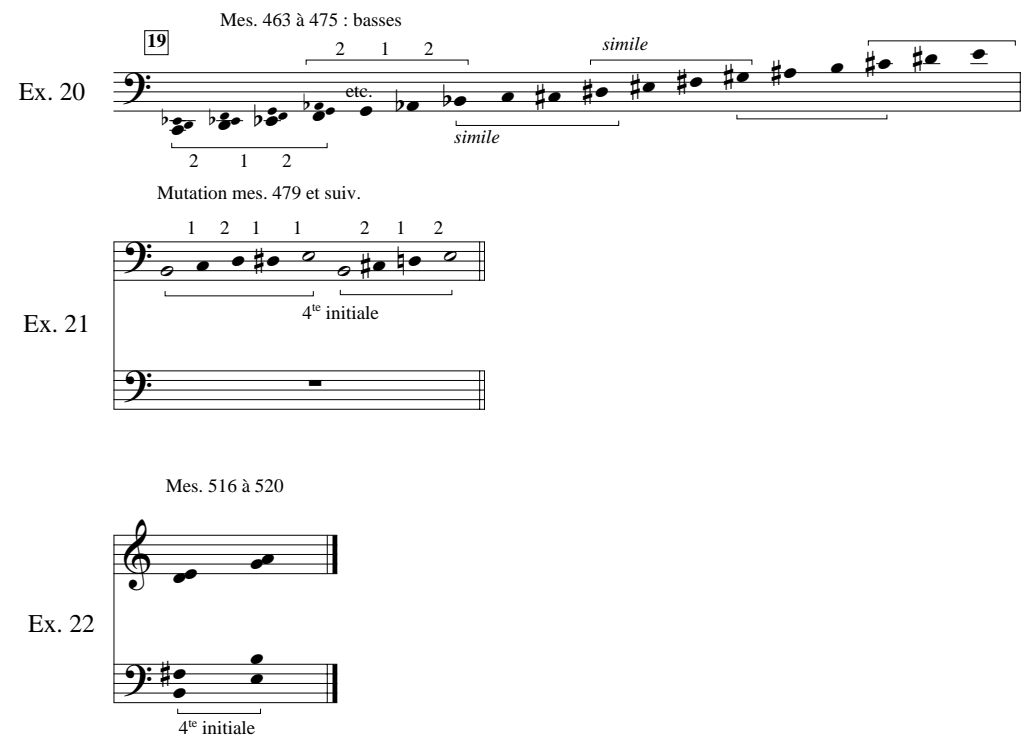